\title{
Quantitative electromyography: a new analogue technique for detecting changes in action potential duration
}

\author{
A. MOOSA AND B. H. BROWN \\ From the Department of Child Health, University of Sheffield, \\ and Regional Medical Physics Department, Sheffield
}

SUMMARY An automatic method using an analogue analyser is described for obtaining an index which is based on the mean phase duration of an EMG signal. This method gives similar values for motor unit action potential duration to an alternative digital method of analysis based on the distribution of time intervals between zero crossings of the EMG signal. The analysis has bee found to give good discrimination of normal and myopathic EMG signals.

An electromyographic signal (EMG), obtained from a concentric needle electrode, consists of a summation of individual action potentials from several of the motor units of the muscle. In diseased muscle the amplitude and waveform of the individual action potentials are often changed and so present an obvious approach to the diagnosis of various diseased states. However, the practical problems of measurement are considerable as the individual action potentials cannot usually be distinguished in the total signal.

Many methods of characterizing normal and abnormal signals have been tried with varying degrees of success. One of the parameters which has been found particularly useful, especially in detecting carriers of Duchenne muscular dystrophy, is the duration of the action potentials which make up the waveform. These durations have been measured manually (Buchthal, 1957; Bosch, 1963; Davey and Woolf, 1964; Hausmanowa-Petrusewicz, Prot, Niebroj-Dobosz, Emeryk, Slucka, Hetnarska, Bendarzewska, and Pucek, 1965; Gardner-Medwin, 1968), and also automatically by detecting the zero crossings or turning points of the signal (Rose and Willison, 1967). However, the manual measurements are extremely time consuming, and the automatic methods are expensive in terms of equipment and can give misleading results, since small noise deflections from the baseline cannot always be distinguished from the action potentials.

A simple analogue computer technique has been developed which is able to detect changes of duration and has been found to give excellenit discrimination between normal and abnormas electromyographic patterns.

\section{METHODS}

Electromyography was performed on 35 childep referred to the Neuromuscular Clinic for invest tion. In 20 of these the diagnosis of myopathy was made on the basis of clinical picture, raised creatin kinase levels, and muscle biopsy (10 had Duchenne muscular dystrophy, four limb girdle dystrophy, two dermatomyositis, three congenital myopathy, an $\Phi$ one dystrophia myotonica). In the other 15 children all investigations were negative and they served as the control group.

EMG signals were recorded, using Disa concentrie needle electrodes (type 9013K0511), from the gastros cnemius, quadriceps, deltoid, and biceps muscles and samples were obtained from several points in each muscle. The EMG was recorded at maximung strength of contraction as far as this was possible i花. children, but no fixed load was used.

The EMG signal was amplified using a Tektronix plug-in (type 2A61) amplifier, and recorded on mago netic tape. It was then analysed automatically using an analogue analyser connected to the recorder i i the replay mode (see Appendix for details of th8 analyser). The analyser could also be connected in parallel with the recorder to give immediate results which were found to be within $\pm 5 \%$ of those obs tained using the recorder in the replay mode.

The output of the analogue analyser was displayee on a chart recorder and gave a value $\psi$ which reprem sents the reciprocal of the amplitude weighted mean ${ }^{+}$ phase duration of the EMG signal (see Appendix fo꽁 
theoretical derivation of $\psi$ ). This value is closely related to the value $\phi$ used by other workers in carrier detection. This value is the ratio of the mean number of phases per potential to the mean potential duration measured manually - that is, it is the reciprocal of the mean phase duration. The value $\psi$ differs from $\phi$ in that it is weighted according to the relative amplitude of the EMG signals, but is obtained automatically.

The integrators in the analogue analyser had a two second time constant and so the value $\psi$ displayed was an integrated value for the previous few seconds. The complete unit was calibrated using a sine wave input of known frequency. As the output from the divider is independent of the amplitude of the sine wave, a calibration directly in terms of frequency was possible.

The index $\psi$ was found for each muscle sample point. The mean value for all the points sampled and the maximum value for any one point was obtained.

Strict comparisons of the values of $\psi$ with other measurements of durations are difficult, as $\psi$ gives a duration which is weighted according to the relative amplitude of the signal deflections (see Appendix). However, comparisons were made with measurements of the distribution of time intervals between zero crossings, as measured using a digital computer (Biomac 1010) and with values of durations quoted by other workers from manual measurement of records.

\section{RESULTS}

It was found that, while the EMG recorded from different points in a muscle gave values for $\psi$ varying by up to $20 \%$, the values obtained for one point were largely independent of the strength of contraction of the muscle (Fig. 1). This was surprising, as at high contractions the action potentials from different motor units of the muscle overlap and it was expected that this would change the mean duration of the negative and positive deflections of the signal.

Figure 2 shows the time interval histogram and the mean duration of the intervals between zero crossings for a single normal EMG. The analogue analyser gave values of $\psi$ for this same record corresponding to a mean duration of $2.1 \mathrm{msec}$. Agreement between the two methods is good in this case and was found to be so in most normal signals. The mean duration of the intervals between zero crossings was calculated for all the 35 patients. The correlation coefficient between these durations and the results, using the simple analogue method, was $0.83(\mathrm{p}<0.001)$. There was disagreement in a number of abnormal records, particularly when there were large changes in the size of the potentials in the EMG. As the analogue analyser gives a mean duration which is amplitude weighted, these differences are to be expected.

A mean normal value for the index $\phi$ quoted by other workers (Gardner-Medwin, 1968) derived from manual measurement of durations is $400 \mathrm{sec}^{-1}$, corresponding to action potentials of duration $7.5 \mathrm{msec}$ and three phases per potential. The index $\psi$ used here gave a value of $470 \mathrm{sec}^{-1}$

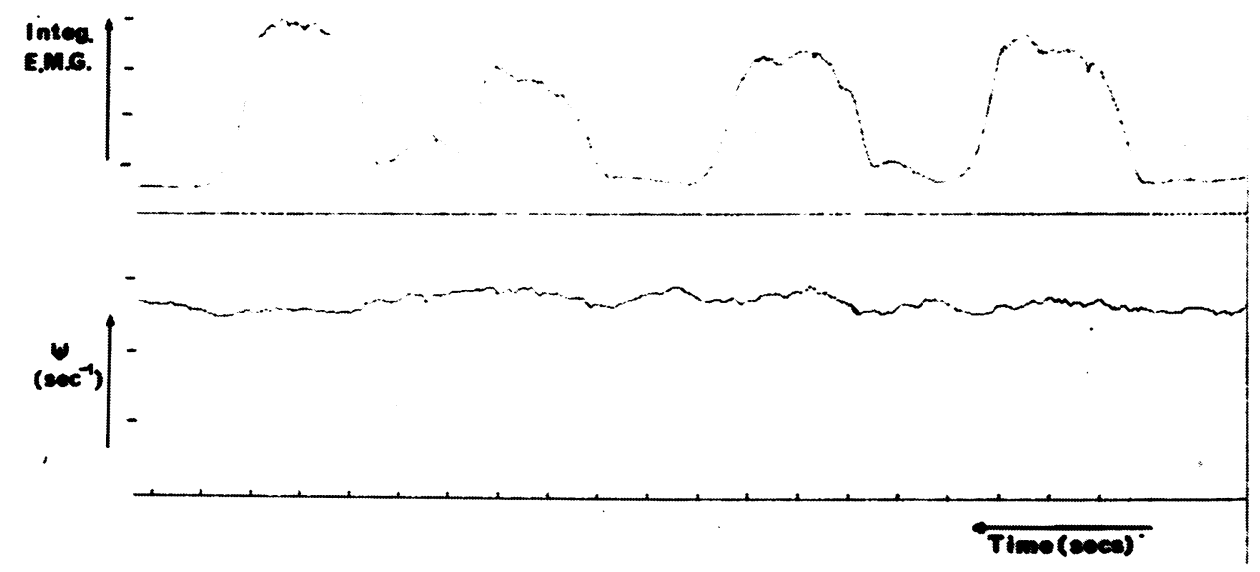

FIG. 1. This shows the small change in the index $\psi$ when the EMG is changed by varying the contraction of the muscle. The EMG is shown after rectification and integration (TC, one second). 


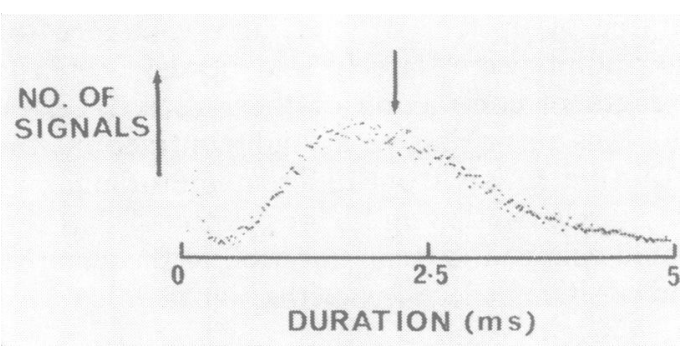

FIG. 2. A histogram showing the intervals between the zero crossings of a normal EMG signal. The arrow marks the mean of the measured durations.

for a control group of 15 subjects and is thus in reasonable agreement.

The mean and maximum values for $\psi$ in the myopathic and control groups are compared in Table 1 and plotted in Fig. 3. The discrimination between the two groups is very good, the values for $\psi_{\max }$ being different at a significance level better than $0.1 \%$. The range of $\psi_{\max }$ in the control group is $428-656 \mathrm{sec}^{-1}$ and if values outside these ranges are taken as abnormal then $75 \%$ of the myopathic group are identified as abnormal.

TABLE

MEAN AND MAXIMUM VALUE FOR $\psi$ IN PATIENTS WITH MYOPATHY AND CONTROL SUBJECTS

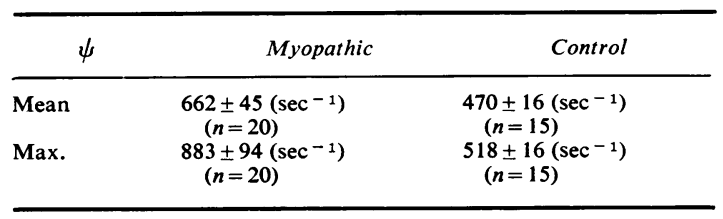

The $25 \%$ of the myopathic group which are not identified as giving $\psi_{\max }$ above the maximum of the control group do not have $\psi_{\max }$ values on the upper side of the normal mean. This is well shown in Fig. 3 which suggests that these five cases might well be a separate group, but clinically they were heterogeneous with three cases of Duchenne muscular dystrophy, two of whom were identical twins, one case of congenital muscular dystrophy, and one case of Becker-type muscular dystrophy.

The results given have not been corrected for the age of the patients. While there is a known increase of motor unit action potential duratior with age, the specification of normal limits of $\sqrt{5}$ at various ages will require a very much greatef number of control subjects than the presente series.



FIG. 3. The distribution of results in the myopath $\vec{E}$ and control groups. Both the maximum and meat values of the index $\psi$ are shown.

\section{DISCUSSION}

It is unlikely that any one index derived from an. EMG will enable an accurate diagnosis to be made of a primary muscle disease. In practice many features of a signal need to be consideref However, of the various quantitative measures ments made to date, the index $\phi$, the reciprocal. of the mean phase duration, appears to be valuable index.

Previous methods of obtaining $\phi$ have bean time consuming because of the necessity t measure individual motor unit action potentiafs and to obtain control records in order to elimif ate subjective variations. The index used in thes work is closely related to $\phi$, being the reciprocad 
of an amplitude-weighted mean phase duration but, unlike $\phi$, it can be determined automatically. The analyser can be connected on line to the EMG recorder and gives an immediate value. As the derivation is such that $\psi$ is nearly independent of action potential repetition frequency, and so of contractile force of the muscle, the method is particularly suited to measurements on children who cannot be asked to produce given muscle contractions. This analogue method of measuring motor unit action potential durations has been shown to give values similar to the duration of action potentials as determined by other workers making manual measurements and to an alternative digital method of analysis. In clinical practice the method of analysis has been found to give good discrimination of normal and myopathic EMG signals.

The usefulness of this method in detecting carriers of Duchenne muscular dystrophy is currently being investigated.

We wish to thank Dr. V. Dubowitz for his interest and encouragement, Mr. D. W. Graham for his technical assistance, and the Regional Medical Physics Department for use of their facilities. The work has been supported by grants from the Muscular Dystrophy Group of Great Britain.

\section{REFERENCES}

Bosch, J. van den (1963). Investigations of the carrier state in the Duchenne type dystrophy. In Proceedings of the Second Symposium on Current Research in Muscular Dystrophy, 1963, pp. 23-30. Pitman Medical: London.

Buchthal, F. (1957). An Introduction to Electromyography. Gyldendal: Copenhagen.

Davey, M. R., and Woolf, A. L. (1964). The electromyographic detection of carriers of muscular dystrophy. Electroencephalographic and Clinical Neurophysiology, $17,705$.

Gardner-Medwin, D. (1968). Studies of the carrier state in the Duchenne type of muscular dystrophy. 2. Quantitative electromyography as a method of carrier detection. Journal of Neurology, Neurosurgery, and Psychiatry, 31, 124-134.
Hausmanowa-Petrusewicz, I., Prot, J., Niebroj-Dobosz, 1., Emeryk, B., Slucka, C., Hetnarska, L., Bendarzewska, B., and Pucek, Z. (1965). Investigations of healthy relatives of patients with Duchenne type dystrophy. In 8th International Congress of Neurology, Vienna, 1965. Abstracts of Papers. Excerpta Medica International Congress Series No. 94, (Abstr.), 210, Excerpta Medica Foundation: Amsterdam.

Rose, A. L., and Willison, R. G. (1967). Quantitative electromyography using automatic analysis: studies in healthy subjects and patients with primary muscle disease, Journal of Neurology, Neurosurgery, and Psychiatry, 30, 403-410.

\section{APPENDIX}

THEORY The electromyographic signal (EMG) which we wish to analyse is a signal such as drawn in Fig. 4 and is made up of discrete waveforms of various durations $\tau_{n}$ and having various numbers of positive and negative deflections, $S_{n}$. Other workers have measured the following:

The mean number of deflections

$$
=\frac{S_{1}+S_{2}+S_{3}+\cdots+S_{N}}{N}
$$$$
\text { The mean duration }=\frac{\tau_{1}+\tau_{2}+\tau_{3}+\cdots+\tau_{N}}{N}
$$

The ratio of these two was used by Van den Bosch (1963) and given the symbol $\phi$, thus:

$$
\begin{array}{r}
\phi=\frac{S_{1}+S_{2}+S_{3}+\cdots+S_{N}}{\tau_{1}+\tau_{2}+\tau_{3}+\cdots+\tau_{N}} \\
=\frac{1}{\text { mean duration of the deflections }}
\end{array}
$$

Bosch (1963) obtained $\phi$ by manual measurement. Any automatic method of analysis needs to be able to distinguish between action potentials and small deflections caused by noise. One way in which to do this is to weight the mean duration according to the amplitude of the deflections. Thus, instead of the index $\phi$ defined by equation (1) we may obtain:

$$
\psi=\frac{\sum_{n=1}^{n=N} a_{n}}{\sum_{N=1}^{n=N} a_{n} \tau_{n}}
$$





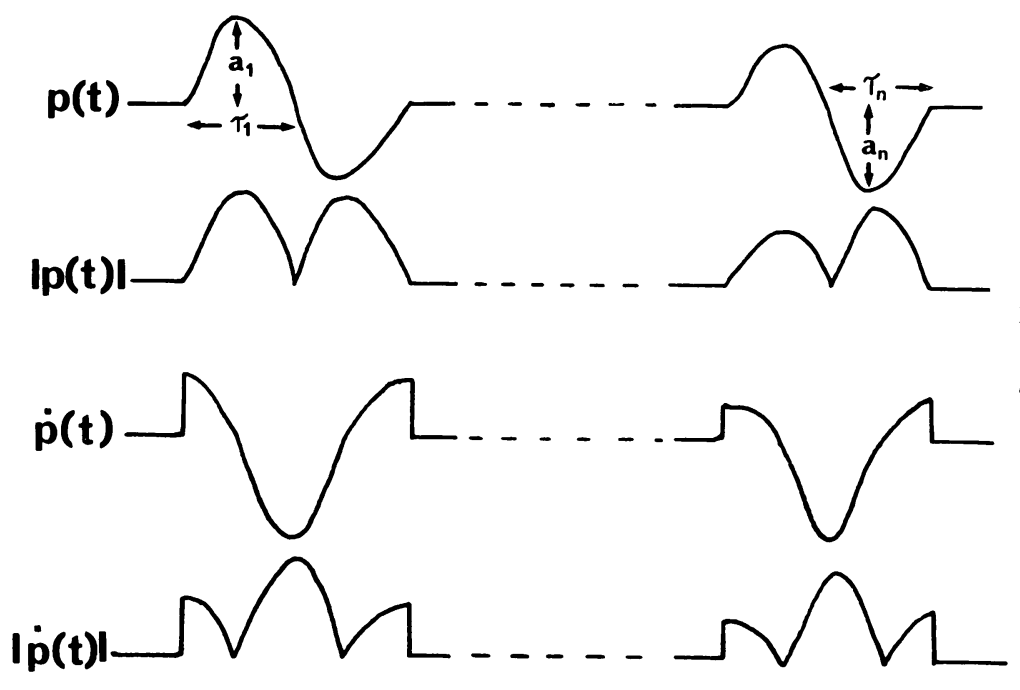

FIG. 5. $p(t)$ is an EMG signal The notation used in the derivas? tion of $\psi$ is shown.

where $a_{n}$ and $\tau_{n}$ are the amplitude and duration respectively of the $n$th deflection of the waveform. When $a_{n}$ is constant we find that $\psi=\phi$.

If we consider the EMG to be apprcximated to the form shown in Fig. 5 and term this $p(t)$, which consists of positive and negative deflections of various amplitudes and durations but an assumed sinusoidal form, then we can show:

$$
\int_{0}^{\mathrm{T}}|p(t)| d t=\sum_{n=1}^{\mathrm{n}=\mathrm{N}} \frac{2}{\pi} a_{n} \tau_{n}
$$

and

$$
\int_{0}^{\mathrm{T}}|\dot{p}(t)| d t=\sum_{\mathrm{n}=1}^{\mathrm{n}=\mathrm{N}} 2 a_{n}
$$

and so on substituting into equation (2):

$$
\psi=\frac{\int_{0}^{\mathrm{T}}|\dot{p}(t)| d t}{\pi \int_{0}^{\mathrm{T}}|p(t)| d t}
$$

Thus $\psi$ may be determined by evaluating this equa- tion using a relatively simple analogue compute such as shown diagrammatically in Fig. 6.

The major assumption in this analysis is that the various negative and positive deflections are sinue soidal in form. This is obviously an approximation. and can only be justified in that the results of this means of analysis are comparable with those fothic. both by manual measurement from the recordings and with measurements of the distribution of crossings made by using a small digital computer.

The circuit designs used to evaluate $\psi$ from equao tion (5) are conventional and use operational amplis fiers in deriving the four stages of differentiation integration, taking the modulus, and division. The output from the analogue divider becomes subject t $\Theta$ increased errors at low input voltages and for thi $\overrightarrow{\mathcal{E}}$ reason a large constant was added to the denomi nator input of the divider when the input signal fe $\mathbb{F}$ below a fixed level. Thus rather than giving an in accurate measurement at low signal inputs the analyser reads zero below a fixed input level.

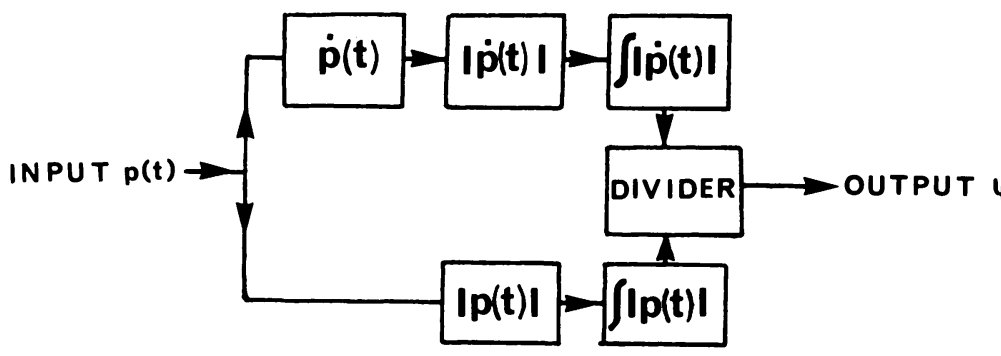

FIG. 6. A block diagram show ing the system used to evaluato the factor $\psi$ from the EMG signaho $p(t)$. 